\title{
Social-Aware Resource Allocation Based on Cluster Formation and Matching Theory in D2D Underlaying Cellular Networks
}

\author{
Wenqin Zhuang ${ }^{1,2}$, Mingkai Chen ${ }^{1,2}$, Xin Wei ${ }^{1,2^{*}}$, Haibo $\mathrm{Li}^{1,2}$ \\ ${ }^{1}$ Key Lab of Broadband Wireless Communication and Sensor Network Technology, Ministry of Education, \\ Nanjing University of Posts andTelecommunications, Nanjing, China \\ ${ }^{2}$ National Engineering Research Center for Communicationand Network Technology, \\ Nanjing University of Posts andTelecommunications, Nanjing, China \\ zhuangwq@njupt.edu.cn, mkchen@njupt.edu.cn,xwei@njupt.edu.cn, lihb@njupt.edu.cn \\ *Corresponding author: Xin Wei \\ Received October 24, 2019; revised December 13, 2019; accepted February 24, 2020; \\ published May 31, 2020
}

\begin{abstract}
With the appearance of wireless spectrum crisis in traditional cellular network, device-to-device (D2D) communication has been regarded as a promising solution to ease heavy traffic burden by enabling precise content delivery among mobile users. However, due to the channel sharing, the interference between D2D and cellular users can affect the transmission rate and narrow the throughput in the network. In this paper, we firstly present a weighted interference minimization cluster formation model involving both social attribute and physical closeness. The weighted-interference, which is evaluated under the susceptible-infected(SI) model, is utilized to gather user in social and physical proximity. Then, we address the cluster formation problem via spectrum clustering with iterative operation. Finally, we propose the stable matching theory algorithm in order to maximize rate oriented to accomplish the one-to-one resource allocation. Numerical results show that our proposed scheme acquires quite well clustering effect and increases the accumulative transmission rate compared with the other two advanced schemes.
\end{abstract}

Keywords: Device-to-device(D2D) communication, social network, matching theory, resource allocation 


\section{Introduction}

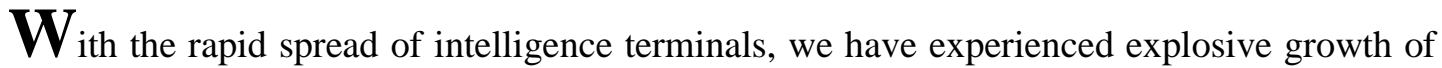
communication capacity in wireless system especially in the past ten years[1]. Naturally, the fact poses a serious challenge to the improvement of local area services. Traditional cellular communication can not meet the emerging demands in future. As the key solution in 5G network, device-to-device(D2D) communication can achieve high-rate transmission of reusing licensed spectrum resource from cellular communication without accessing in base station[2]. It can both ease the traffic burden and enhance the spectrum utilization. Thanks to these advantages, D2D communication has gained more attention in the investigation of local area service[3].

Despite the fact that D2D communication can improve the system performance by reusing licensed spectrum resource, the resource allocation for the network also influences the system performance adversely. Therefore, how to ensure the transmission need of different D2D links is a quite significant project in resource allocation. Several schemes were discussed in the previous work. Xu et al. accomplished resource allocation based on implementing a reverse iterative combinatorial auction [4]. Nguyen et al. improved the effect of resource allocation by adjusting the transmission power and removing the interference by pricing scheme [5]. These studies either focus on increasing the throughput of the network or minimize the mutual interference. However, they are lack of consideration about the influence from the social ties between mobile users [6] [7]. Since the device does not signify as a smart phone, it also means the manners of mobile users share resource in the network.

It is seen that social connection among mobile users in D2D communication can severely affect transmission performance and provide the motivation that users may assist each other. From the aspect of offloading, social-aware approach is quite different from a conventional context-unaware approach [8][9]. Therefore, how to leverage the social characteristics plays a key role on designing effective resource allocation proposal. And maximizing energy-efficiency can be formulated between D2D and cellular users(CUs) by considering their priorities based on social-aware virtual MAC protocol [10]. In the dynamic situation, relay selection and management are reviewed with social awareness-aided spectrum-power trading in cooperative D2D communications [11] [12]. Moreover, social-aware user connection performance is integrated by the average link rate and the social reciprocity, and stimulates mutual cooperation to ensure D2D content sharing [13]. However, none of these literature mentions how social attribute to impact on the access of D2D communication. And it is a significant issue to maximize the joint social and physical benefits in the network.

Based on the crucial problem mentioned above, we attempt to combine social ties and physical connection to optimize performance of the network, especially the social-aware throughput. Therefore, in this paper, our contribution is that we combine the social and physical network to highlight the influence of social-tier on resource allocation among users in D2D networks. Firstly, in order to jointly consider the social attributes and physical proximity to D2D underlay communication network, we measure the social graph and physical graph quantitatively and propose a weighted-interference minimizing oriented cluster formation scheme to guarantee the system performance. Next, inspired by the matching theory [14] [15], we propose a stable rate-maximizing based on matching algorithm to accomplish the one-to-one matching between cellular users and D2D pairs. Finally, the result demonstrates 
that our proposed scheme acquires quite well clustering effect and increases the overall transmission rate greatly compared to the other leading-edge schemes.

The reminder of this paper is organized as follows. Firstly, we have listed the related work in this field and classified to the different types, in Section II. We introduce the system model in Section III. Then, we propose our weighted-interference minimizing oriented cluster formation scheme and stable rate-maximizing based matching algorithm in Section IV. Then, in Section V, Simulation result and analysis are given, and finally we conclude the paper in Section VI.

\section{Related Work}

We survey the literature on social-aware D2D communication, such as social content sharing, social-aware resource allocation, social-aware relay selection.

Social Content Sharing. Social network has tremendously changed content sharing. In this network, every user equipment(UE) is smart to determine which content to provide or cache and to share their own social characteristics. By taking wireless features, social characteristics and device intelligence into account, these works formulate an optimization problem by integrating power control, mode selection, mobility and incentive mechanism to achieve a proactive caching or content replication [13], [16]-[19]. All contents are managed in content-centric mode, where users can send their interests to obtain content with naming information, resulting in a reduction of network traffic load [17]. Perhaps, the authors introduce the notation of social selfishness from social knowledge to represent the social-aware rate and achieve an effective cooperation together [19]. However, the social content sharing does not consider the transmission issue in the D2D communication. They all assume the D2D communication can initiate without influence of the physical layer all based on social characteristics, which are not actual.

Social-aware Resource Allocation. The existing social-aware resource allocation approaches have considered physical domain constraints and social domain factors, which should result in effective D2D links among users' willing to share interests, which are called social-aware resource allocation. Moreover, knowledge of social characteristics derived from social network may play a key role in D2D multicast by sufficiently utilizing both the physical and social properties of users [20]. Due to the user selfishness, the authors can use the game theory to maximize the user's utilities and decide whether to opportunistically share the content with another user [21]. In [22], the authors discuss the domain of D2D communication with respect to social-aware resource allocation and optimization. Then, the authors devise a taxonomy and outline the key requirements to enable social-aware resource allocation. And then, some literature [23] [24] has debated the social-aware resource allocation between D2D communication and communities. They exploit social ties to enhance D2D resource sharing or channels sharing among a group of people.

Social-aware Relay Selection. Employment of social-aware D2D relay communications also has been studied in amounts of literatures. In [25], the authors formulate a problem of social-aware D2D relay selection and propose a two-stage D2D selection scheme. Moreover, the relay selection is related to energy efficiency and mobility [26] [27], which exploits social ties in human social networks to enhance cooperative D2D communications. Moreover, leveraging social trust, the researchers also focus on social-aware energy-efficient relay selection mechanism, which gives the reason why the user serves the other users in the network. 
Summary. All of these research are built on social network and interference control. For example, content sharing establishes on a stable relationship and reliable transmission link. Resource allocation serves for the reliable transmission link and also reduces the interference between the links. Relay communication may bring more D2D interference in the network, but we should cut down the influence of this issue. Since interference control is the basis of these studies, we should bring the social attributes into channel control in order to reduce the interference in the transmission. Hence, in this paper, we focus on the social-aware interference control to solve the problem.

\section{System Model}

\subsection{System Overview}

We consider a D2D-assisted small cell network (SCN), consisting of random-located access point (AP) and $C$ mobile users, denoted as the set of $\left\{C_{c} \mid c \in 1,2, \ldots, C\right\}$. Mobile users which are in physical and social proximity form as $D$ D2D pairs $\left\{D_{d} \mid d \in 1,2, \ldots, D\right\}$. These D2D pairs can implement D2D communication by reusing the licensed spectrum resource for traditional cellular communication. Besides, there are a set of orthogonal subcarriers for uplink transmission which are labelled as $B_{k},\left\{B_{k} \mid k \in 1,2, \ldots, K\right\}$, and D2D transmitter can choose power level from the set of $\left\{P_{l} \mid l=1,2, \ldots, L\right\}$. In our investigation, we are tending to depict the content sharing mechanism with two-dimension architecture in details as illustrated in Fig. 1. As shown in Fig. 1, we know the system includes the two different domains, social domain and the physical domain. The social domain can lead the physical domain to alter and optimize the performance in the physical layer. It may modify the related parameters, like power and channel, to satisfy the social requirements.

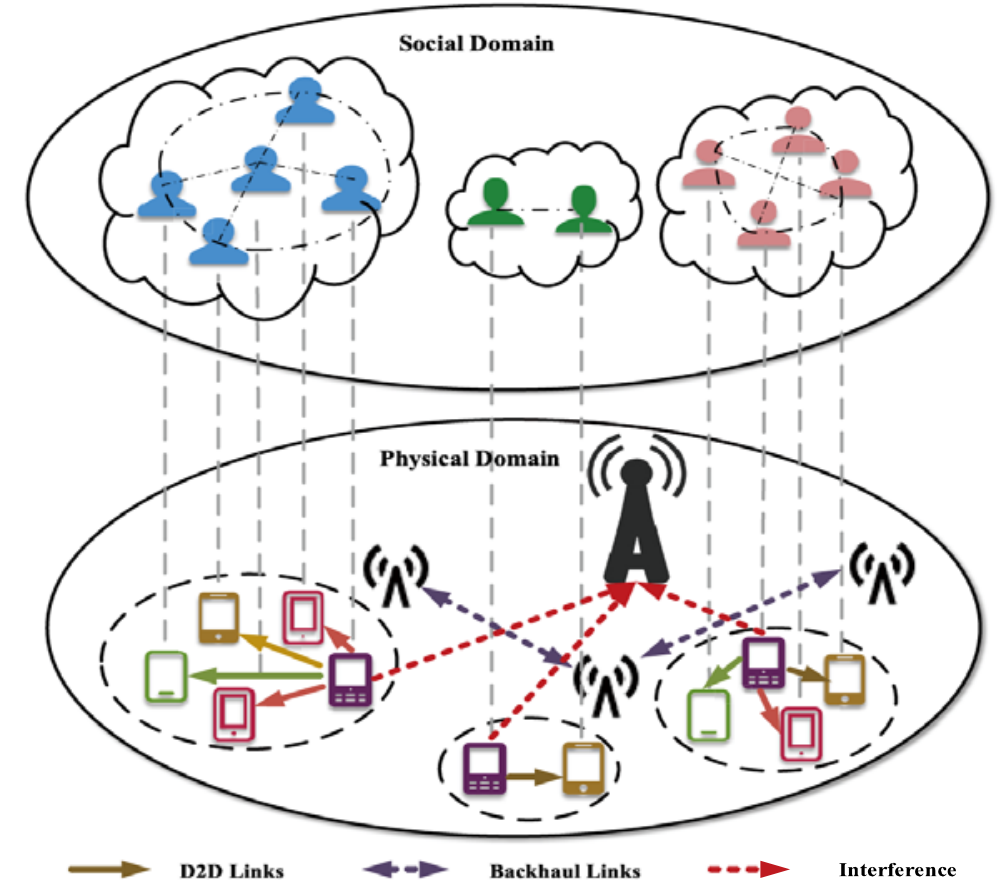

Fig. 1. Illustration of social-aware D2D system 


\subsection{Interference Graph Formation}

In the physical domain, base station (BS) connects to the AP via a high speed wired link or backhaul link. AP can acquire numerous data resources from BS and easily deliver to mobile users via cellular communication. It is said that if AP and all mobile users are perfectly synchronized the users can access to core network with a very high speed. There exist two kinds of wireless data transmission path in the SCN: the ordinary paths between the source AP to the destination mobile users and the D2D path between condition-enabled users. For the reason of spectrum reusing, unbearable interference is induced into network inevitably. Besides, the degree of interference derives from proximity between users. The closer users geographically are, the more severe interference they would mutually cause. Therefore, we depict the interference graph depending on the network topology.

We consider a typical scene consisting of cellular communication link and D2D communication link dominated by the AP. We construct an undirected weighted interference graph $g_{a, b}^{i}=\left(N, \varepsilon_{a, b}^{i}\right)$. Thereinto, the vertex set $N$ denotes arbitrary cellular links or D2D links in the cell, the edge set $\varepsilon_{a, b}^{i}$ denotes the weighted potential mutual interference between the two vertices $a$ and $b$. To simplify the interference graph, we assume that BS assigns equal transmitted power $p_{\mathrm{t}}$ for each uplink cellular communication and all the D2D transmitters hold the same maximum power level $p_{\max }$ with efficient energy. Different from the interference graph proposed for resource allocation problem in [17], we have to announce the resource selection list $B_{k},\left\{B_{k} \mid k \in 1,2, \ldots, K\right\}$ firstly. For example, when $k_{i}$ and $k_{j}$ are prone to establish an communication links, an channel state information will be sent to BS, and BS replies with the channel estimation information, then $k_{i}$ calculates signal interference plus noise ratio(SINR) when certain indices of $B_{k}$ is chosen. As a result, the interference link varies depending on the channel response toward different RBs. With more reasonable consideration, we weight the mutual interference between vertex $k_{i}$ and $k_{j}$ as $I_{s}=I_{k_{i}, k_{j}}+I_{k_{j}, k_{i}}$. Note that there is no interference when D2D communication implements on separate channels.

Based on the channel estimation information provided by the BS, we can calculate $I_{s}$ as follows:

$$
I_{s}\left(\mathrm{~B}_{1}, \ldots, \mathrm{B}_{\mathrm{k}}\right)=\left\{\begin{array}{l}
p_{t} g_{k_{i}, k_{j}}\left(\mathrm{~B}_{1}, \ldots, \mathrm{B}_{\mathrm{k}}\right), k_{i} \in C, k_{j} \in D \\
p_{l} g_{k_{i}, k_{j}}\left(\mathrm{~B}_{1}, \ldots, \mathrm{B}_{\mathrm{k}}\right), k_{i}, k_{j} \in D
\end{array} .\right.
$$

\subsection{Social Graph Formation}

On one hand, people in close proximity geographically are more willing to share information with each other. On the other hand, a group of friends, students in the same class or employees in the same company also have common interests. In the social domain, users with high trust can choose his D2D pair to exchange appointed content within the community $S$. Since the mobile users are anonymous and have different interests, it influences how mobile users access to networks. Thus, social attributes have influence on resource allocation strategy greatly. So it is necessary to maintain a record of user's relations with each other. For this 
purpose, we introduce social graph $g_{a, b}^{s}=\left(N, \varepsilon_{a, b}^{s}\right)$ to exhibit the social connection between mobile users where the edge set $\varepsilon_{a, b}^{s}$ denotes the weighted social ties between two vertices $a$ and $b$. The value of social ties reflects the strength of social relationship between users.

For most of realistic social networks which hold explicit weight for a pair of vertices, we define $\varepsilon_{a}=\sum_{b} \varepsilon_{a, b}^{s}$ as total strength of weight of users $a$ and $\omega_{a, b}$ as the transmission possibility of random vertices in the social graph. Inspired by the contact-based epidemic spreading model illustrated in [16], we are tending to depict the content propagation process as a two-state model. It denotes that all of the users in the spreading model are divided into two states: Susceptible (S) and Infected (I). The content sharing mechanism can be summarized as follows: user $a$ initially holds the common interested content shares the content with probability $\omega_{a, b}$ to user $b$ at susceptible state by $\lambda$ trials. In this process, the contact possibility $\omega_{a, b}$ can be expressed as follows:

$$
\omega_{a, b}=1-\left(1-\frac{\varepsilon_{a, b}}{\varepsilon_{a}}\right)^{\lambda} .
$$

With dynamic content sharing processing, we can divide the situation into two different cases from the scope of epidemiological model: the contact process (CP) and the reactive process (RP). The most obvious difference between the two cases is existing only one individual stochastic contact per unit time in CP, while all the users can be candidates to provide the content.

In traditional investigation, the object function is typically modeled as the minimization problem of mutual interference. We use weighted interference to evaluate the influence of social ties on our network. Compared to the average occasion, D2D links with high contact probability $\omega_{a, b}$ implicit heavy transmission tasks usually. And it presents a strict requirement for D2D transmitter. Naturally, accumulated interference may bring congestion accordingly. Taking social tier into consideration, we are tending to present a social weighted interference model. In this model, we define the weighted social interference for each cellular-D2D reusing pair expressed as:

$$
I_{n}\left(B_{1}, \ldots B_{k}\right)=I_{s}\left(B_{1}, \ldots B_{k}\right)+\omega_{a, n} I_{a}\left(B_{1}, \ldots B_{k}\right) .
$$

We can easily find that weighted interference consists of two parts: the interference aroused by cellular communication and potential interference induced by the associated D2D links. The latter part is evidently constraint to the contact possibility $\omega_{a, b}$.

\subsection{Problem Formulation}

After building the model of network, we attempt to give the problem that we concern on. We define $g_{d}$ as the channel gains among the D2D links and $g_{c, d}$ as the interference channel gains from $C_{c}$ and $D_{d}$. In addition, $g_{c, A P}$ denotes the interference channel gain between $C_{c}$ and AP and $g_{d, A P}$ denotes the interference channel gain between $D_{d}$ to AP. Thus, SNIR of $C_{c}$ to $\mathrm{AP}$ is 


$$
\Gamma_{a, b}^{C}=\frac{p_{t} g_{c, A P}}{p_{l} g_{d, A P}+N_{0}},
$$

where $N_{0}$ is the noise power of the channel.

Then, SINR of $D_{d}$ in each cluster is

$$
\Gamma_{a, b}^{D}=\frac{p_{l} g_{R(s b}}{p_{t} g_{c, d}+N_{0}} .
$$

Therefore, the achievable transmission rate of cellular user $C_{c}$, the achievable transmission rate of $D_{d}$ whose spectrum resources is provided by the cellular users $C_{c}$ is given respectively as:

$$
\begin{array}{r}
R_{a, b}^{C}=\log _{2}\left(1+\Gamma_{a, b}^{C}\right), \\
R_{a, b}^{D}=X_{c, d} \cdot \log _{2}\left(1+\Gamma_{a, b}^{D}\right),
\end{array}
$$

where $X_{c, d}=\left[X_{c, d}\right]_{C \times D}$ denotes a binary set which records the reuse relationships between the cellular user $C_{c}$ and D2D links $D_{d}$.

Our objective is to maximize the system throughput while guarantee the normal communication demand, so the optimization problem can be formulated as following:

$$
\begin{aligned}
& \arg \max \sum_{i=1}^{C} \sum_{j=1}^{D} R_{i, j} \\
& \begin{array}{c}
\text { s.t } \mathrm{C}_{1}: \mathrm{R}_{\mathrm{i}, \mathrm{j}}=\mathrm{R}_{i, j}^{C}+x_{i, j} R_{i, j}^{D} \\
\mathrm{C}_{2}: 0 \leq p_{l} \leq p_{\text {max }}{ }^{D} \\
\mathrm{C}_{3}: x_{i, j}=\{0,1\} \\
\mathrm{C}_{4}: \sum_{i=1}^{C} x_{i, j} \leq 1, \forall j \in D
\end{array} .
\end{aligned}
$$

where $C_{1}$ indicates that the system throughput is related to the resource allocation strategy and the channel gains of the cellular communication. $\mathrm{C}_{2}$ indicates that the transmission power level of each D2D transmitter is below the maximum value $p_{\max ^{D}} \cdot \mathrm{C}_{3}$ and $\mathrm{C}_{4}$ restrict that one D2D links can reuse spectrum resource from only one cellular user.

\section{Cluster Formation and Matching Theory Based Resource Allocation}

In this section, we tend to solve the optimization problem depicted in (8). To maximize the sum rate of the D2D-enabled cluster, we attempt to propose a reasonable strategy for spectrum allocation based on the cluster formation in SCN. Firstly, we calculate the social and physical attitude of users in the network and divide the user into different cluster. Secondly, we define the different probability of D2D communication as different price. When user launches the certain D2D communication, it should pay equivalent price to borrow the resource block (RB) from CUs. Finally, we should match the D2D users and CU in the same channel in order to maximize the transmission rate, so we can calculate the throughput of the whole network. 


\subsection{Cluster Formation}

For simplicity, we assume there is only one AP in SCN and we also think the AP can only disseminate content toward a single community. The original intention of cluster formation is to avoid the situation that mobile users with strong social connection form high-interference link in SCN. As a result, those links will reduce system throughout greatly. We propose a spectral clustering scheme to refine different communities into several clusters, which involves the following steps. The example in Fig. 2 show the basic idea of the scheme.

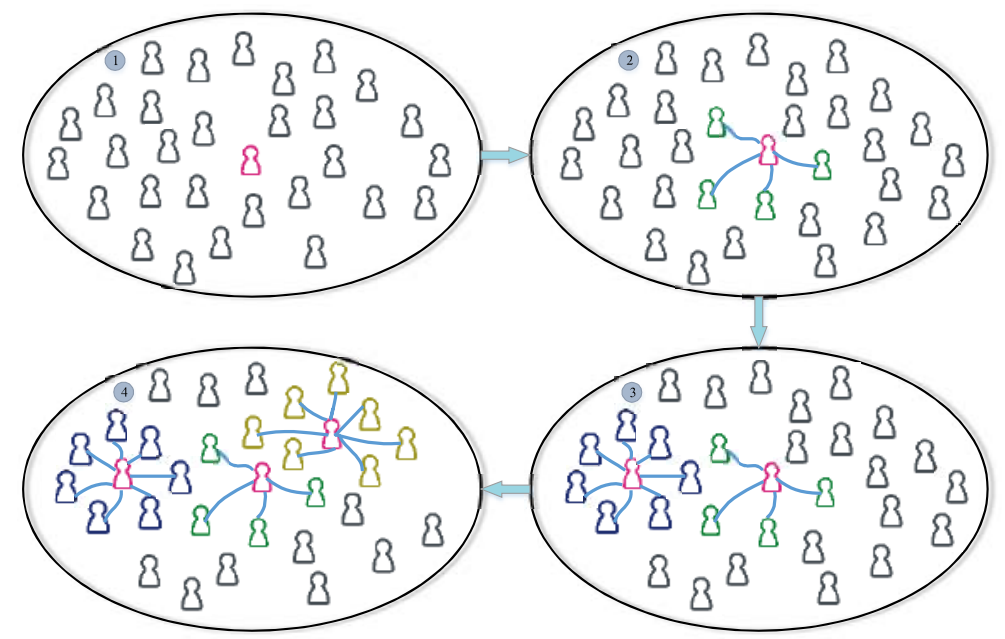

Fig. 2. Illustration of cluster formation

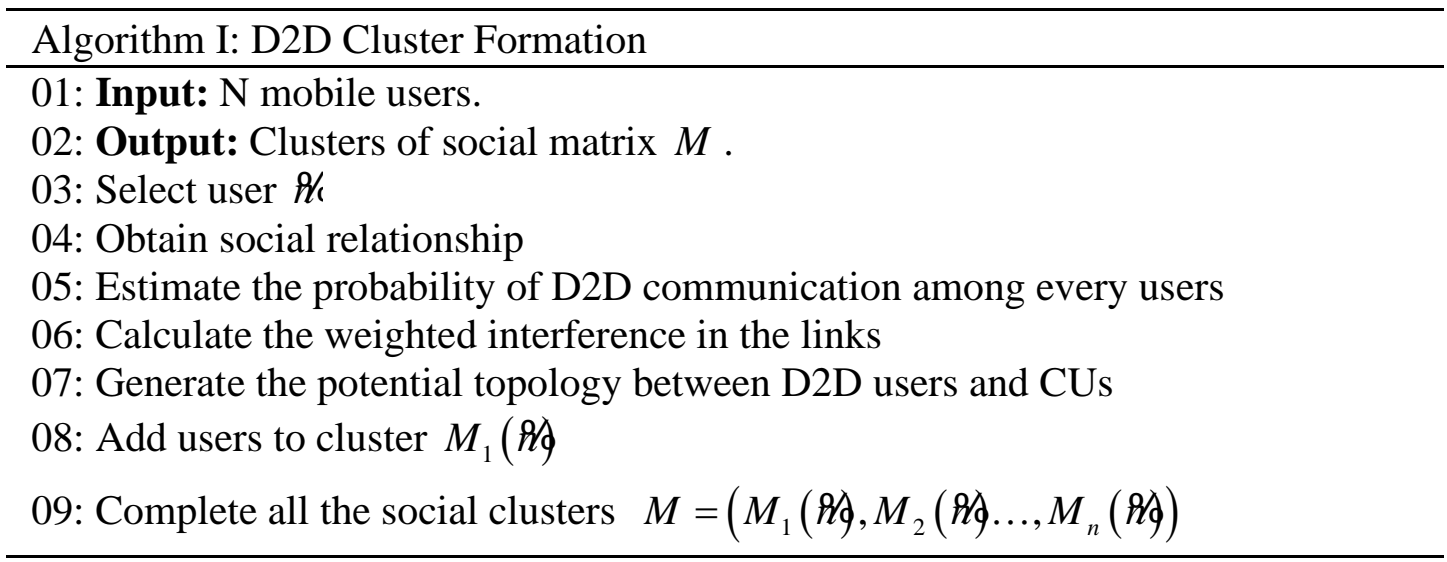

Step 1: For the community $S$, the user $\beta /$ that introduces minimal interference to AP can be selected as the source node in the community if the qualification can be met. That is the total strength of social relationship between users exceed the average level $\varepsilon_{P C}$. When users only have social relationship, they can establish D2D link. It means user trusts the other users so that they can communicate with each other.

Step 2: We define the transition probability matrix as $P_{(1 \times N)}=\left[\omega_{a, b}\right] . \omega_{a, b}$ represents the possibility of the establishment of D2D link between users $a$ and $b$. Steming from the source node $B /$, if and only if the following two conditions are satisfied, the candidate nodes can be 
added into the cluster $M_{1}(B)$. After certain users join the cluster, the weighted interference is below the weighted interference threshold. Otherwise, $M_{1}(B) \in C_{c}$. The searching process ends when all the nodes are traversed.

Step 3: The set $S=S-M_{1}$ ( repeats the operation from Step $1 \sim$ Step 2 among the other nodes until all the social clusters $M=\left(M_{1}(\beta), M_{2}(\beta) \ldots, M_{n}(\beta \not)\right)$ accomplish.

The detail of cluster formation is indicated in Algorithm I.

It is worth to mention that we set restrictions for the cluster formation processing as the number of the final-formed cluster can't exceed $L$ to guarantee $N-2 D \times L \geq D$ in Step 2 . In other words, one D2D pair has at least one resource reusing choice to select according to the restrictions.

\subsection{Price Strategy}

As BS plays as a role of dominator in SCN. When BS assigns a resource block to the D2D pair, the cellular users will suffer interference at the same time. To acquire considerable system throughout and prevent CUs from severe interference, BS should charge the D2D links with different "price" as they competing for the spectrum resource selfishly. In particular, the function for charging price $\gamma$ can be expressed as

$$
\gamma=x+y \cdot B_{k}^{\alpha}, k \in 1,2, \ldots, K
$$

where $x, y, \alpha$ are non-negative constant. And we assume $\alpha \geq 0$ to guarantee the price function convex. Besides, $B_{k}$ represents the sub-bandwidth allocated to the $k-t h$ D2D pair. The aim of establishing price set is to make up the interference caused by D2D transmission.

\subsection{Matching Algorithm}

In this section, we propose a distributed algorithm based on matching theory to resolve the allocation problem. We put forward to basic concept in matching theory firstly. Then we establish the preference set from the perspective of maximizing the throughput. In this process, price strategy is similar to bargaining in game theory, which is introduced to avoid the occurrence of multiple reuse scenarios. At the same time, corresponding algorithm is listed in details.

1) Matching preference establishment

The basic concept of matching theory elaborates the preference correlation of finite set $M=\left(m_{1}, m_{2}, \ldots m_{n}\right)$ towards another finite preference set $W=\left(w_{1}, w_{2}, \ldots w_{n}\right)$. Preference means the priorities of selections while we choose from the set of $W$. The premise definitely exists at least a favorite choice in $W$ for each elements $m_{i}$ in $W$. To illustrate, we assume $m_{i}$ has two alternative selections $w_{1}$ and $w_{2}$ in preference set. If $m_{i}$ prefers $w_{1}$ to $w_{2}$, it means the priorities of $w_{1}$ is higher than $w_{2}$. Learning from the basic concept of matching theory, we can give some definitions in our investigation:

Definition: $T=\left(T_{1}, T_{2}, \ldots, T_{n}\right)$ denotes the set of D2D pairs towards resource block list $B_{k} . T_{i}$ denotes the set of preference selections of D2D pair which can be expressed as $1 \times K$ 
matrix $T_{1 \times K}=\left(T_{1}, T_{2}, \ldots, T_{k}\right)_{1 \times K}, k=1,2, \ldots K$. To sum up, the sum set of all D2D pairs can be expressed as a matrix, which is $0-1$ matrix:

$$
T_{N \times K}=\left(\begin{array}{ccc}
T_{1,1} & \ldots & T_{1, k} \\
\mathrm{M} & \mathrm{O} & \mathrm{M} \\
T_{n, 1} & \mathrm{~L} & T_{n, k}
\end{array}\right) .
$$

As we establish the preference set to maximize throughput, we denotes the rate set is corresponding to the set $T_{i}$ as follows with the premise of all of the constraints:

$$
U=\left(\begin{array}{ccc}
U_{1,1} & \ldots & U_{1, k} \\
\mathrm{M} & \mathrm{O} & \mathrm{M} \\
U_{n, 1} & \mathrm{~L} & U_{n, k}
\end{array}\right) .
$$

It is important to note that we restrict one D2D cluster can reuse the spectrum resources from at most one CU. As a result, the sum of rows and columns of $T_{N \times K}$ should meet the constraints: $\sum_{n=1}^{N} \sum_{k=1}^{K} T_{n, k} \leq 1$. Hence, there are $k$ strategies of spectrum allocation and the element $U_{n}$ is corresponding to the weighted sum rate of each allocation strategies. The preference list of D2D pairs towards different cellular users can be obtained by solving the optimization problem in (8). Back to the matching theory, we define the preference set $O_{N \times K}=\left(O_{1}, O_{2}, \ldots, O_{n k}\right)_{N \times K}$ by descending order. According to the obtained preference set, we propose a matching algorithm based on the Definition above in details.

2) Resource Allocation Matching Algorithm

By exploiting the established preference set, we tend to design a distributed resource allocation matching algorithm. Before that, we consider the situation that several D2D pairs request for the same resource blocks occupied by the same cellular users. Because of the centralized property of BS, it will distribute "price" for different resource band when these bands are requested by different D2D pairs. By taking the pricing operation of AP into consideration, we explain the procedure of our proposed matching algorithm as follows:

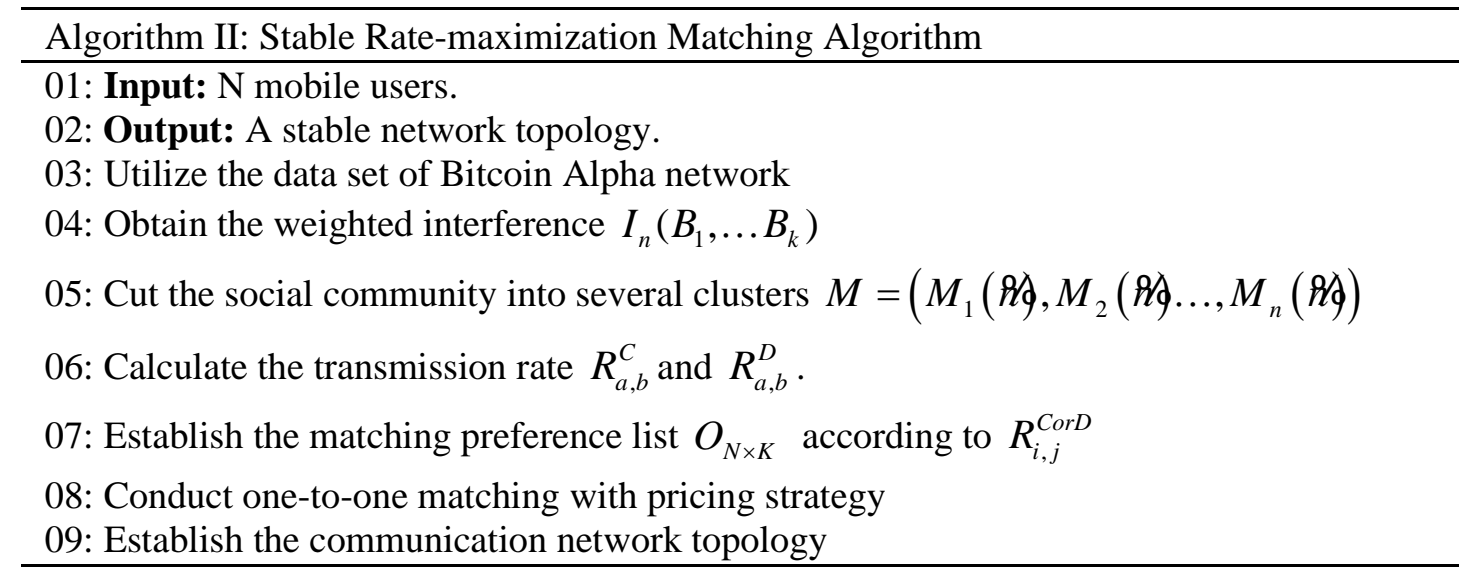


Step 1. First of all, we utilize the concept of price cost to reuse RBs in CUs. Let $\gamma_{n \times k}=\left(\gamma_{n, k}\right)_{n=1, k=1}^{n=N, k=K}$ represents the price cost when D2D pair $k$ reuse RB occupied by the cellular users $n \cdot \gamma_{n, k}$ represents the cost generated by per bit rate associating with AP and bandwidth. The expression of price cost can be acquired from (9).

Step 2. For D2D pairs, each D2D pair can determine its most preferable RBs according to the preference set $O_{N \times K}$. For cellular users $k$, if there exists only one D2D pair which is willing to reuse RB belonging to cellular users $k$, BS will allow the associated link directly. But in more common situation, there exist several requests of D2D pairs for the spectrum resource from the cellular users. By considering the definition of price cost illustrated in Step 1, we can measure the price cost of each request and determine which D2D pair is chosen as the candidate. Each step of iteration results in a CU-D2D pair. The iteration ends when there is no request from D2D pairs or the qualification $N-2 D \times L \geq D$ in SCN.

Step 3. After the operation in Step 1 and Step 2, the output yields to the whole matching decision of CU-D2D pairs. And we based on the decision to establish the communication network topology in the cellular network.

These above steps provide a basic idea of stable rate-maximization matching (SRM) algorithm for our D2D communication underlay cellular network. The detail of the algorithm is elaborated in Algorithm II.

\subsection{Analysis of Stability and Optimality}

In this section, we provide imperative analysis to prove the stability and optimality of our proposed algorithm as an one-to-one matching.

1) Stability

Theorem 1: There always exists a stable matching scheme no matter what the pattern of preference list is.

Proof: According to Definition in Section IV-C, we assume a arbitrary preference list $O_{N \times K}=\left(O_{1,1}, O_{1,2} \ldots O_{n, k}\right)_{N \times K}$, it means cellular user $k$ is the most preferable choice for D2D pairs $n$. Each cellular user which receives more than one resource request will refuses all but its most preferable D2D pairs as candidates. Those rejected in the first stage will send proposal towards their second preferable choice. Then, cellular user chooses their favorite choice among new proposal and candidate derived in the former stage. This process will repeat in the same manner.

In at most $n^{2}-2 n+2$ iterations, cellular user will have received one proposal at least. For the reason that one D2D pairs can only request for the resource from the same cellular user no more once, every cellular users will ends in an CU-D2D pairs in peri time. If $n<k$, the matching process will end when all of the cellular users have been requested. If $n>k$, it will end when all of D2D pairs have been chosen for candidates or been rejected by the cellular users. Thanks to the symmetry of the candidate of users, it will bring out a stable set of matching list in the end.

2) Optimality

Theorem 2: Every stable matching assignment is optimal under the SRM algorithm.

Proof: Assuming that in certain point, cellular users $c_{1}$, having received several request from a set of better-qualified candidate $d_{1}, d_{2} \ldots, d_{\alpha}$, but reject D2D pairs $d_{\beta}$. In such 
situation, we can conclude that each $d_{i}$ prefers $c_{1}$ than all the others and $c_{1}$ prefers each $d_{i}$ than $d_{\beta}$. Note that we denote $d_{i}$ as $d_{\beta}$ and $d_{i}$ respectably. In addition, we have to request for a less preferable cellular user for spectrum resource. But this assignment is not stable for the reason that it can not benefit the throughput at the utmost extend. Hence, the assignment is unstable and $d_{\beta}$ can not establish link with $c_{1}$. The assumption shows that our matching algorithm only refuses the request which is impossible to be admitted into a stable assignment. So the result of assignment is always optimal.

\section{Simulation Results and Discussion}

In this section, we provide the simulation results to illustrate the performance of our proposed SRM algorithm. Main parameters have been given in Table 1. In physical domain, the simulation scenario is restricted within a $500 * 500 \mathrm{~m}$ area. The radius of D2D communication is $50 \mathrm{~m}$. The channel fading is -20 . And the noise power is $9 \mathrm{dBm}$ at each user device. The power of transmission is $23 \mathrm{dBm}$. Moreover, the interference threshold is $5.6 \times 10^{-6} \mathrm{~W}$, which means the communication interrupt when interference is higher than this value[29]. In social domain, we utilize the explicit weighted signed directed network Bitcoin Alpha to evaluate the trust degree between the nodes. All of the nodes are distributed randomly in the coverage of the physical domain. Both path loss model and shadow fading are taken into account in cellular and D2D links. Utility function maximization(UFM) and optimal social-community aware resource allocation(OSRA) are used for comparison [30].

Table 1. Applications in each class

\begin{tabular}{|c|c|}
\hline Parameter & Value \\
\hline \hline Coverage radius of BS & $500 \mathrm{~m}$ \\
\hline Distance of D2D & $50 \mathrm{~m}$ \\
\hline$\alpha$ & 20 \\
\hline Noise power & $9 \mathrm{dBm}$ at device \\
\hline Transmission power & $23 \mathrm{dBm}$ \\
\hline Interference threshold & $5.6 \times 10^{-6} \mathrm{~W}$ \\
\hline
\end{tabular}

Fig. 3 shows the result of the cluster formation based on the spectrum clustering algorithm. From the result, we can observed that mobile users which satisfy the requirement of minimizing the weighted interference can form the cluster to exchange information directly. Thanks to the reason for considering the social and physical factor synthetically, we find proximity users geographically will not be classified into the same cluster in some situation. In certain degree, it reflects the reliability of our proposed cluster formation algorithm. 


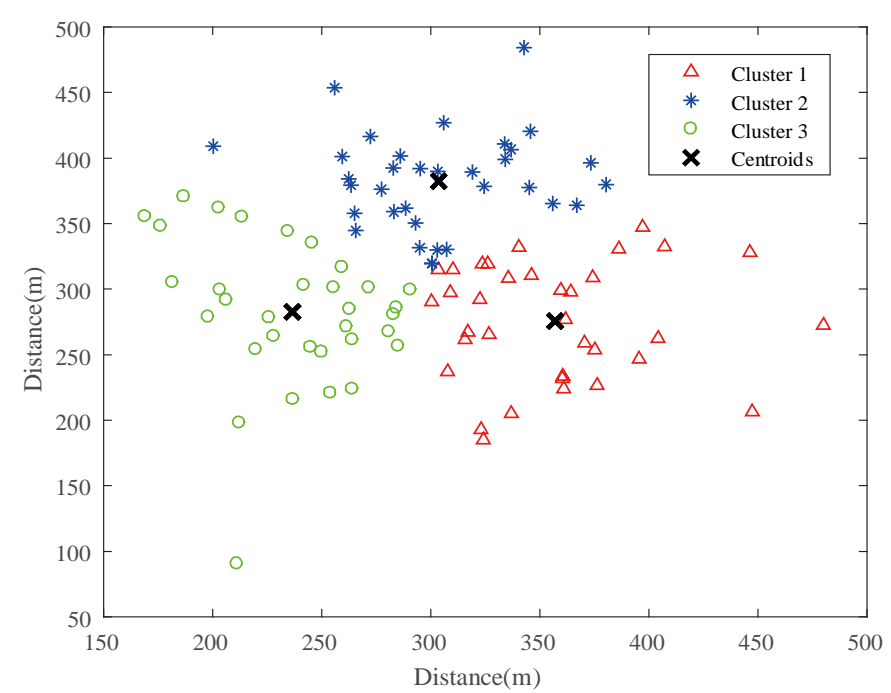

Fig. 3. Result of cluster formation

Fig. 4 and Fig. 5 compare the sum rate of the three algorithm under the isolated scenarios, in which D2D communication can only implement within the same cluster. We set the number of cluster as $N=3$ and clustering coefficient as $\sigma=0.1$. In the network, we cut the association between different cluster to avoid the appearance of interference-unbearable link, we can observe that significant performance enhancement can be achieved when cellular users are relatively sufficient. But when we change the weighted-interference threshold, the number of D2D pairs changes accordingly. When cellular users are not sufficient, performances of SRM and UFM are roughly equal. The reason is that it lacks enough cellular users to provide sufficient spectrum resource, when the threshold is easy to meet. Hence, the interference within the network increases dramatically. As a result, the advantage of our proposed algorithm is not evident. But in general, the proposed scheme outperforms than the other two averagely.

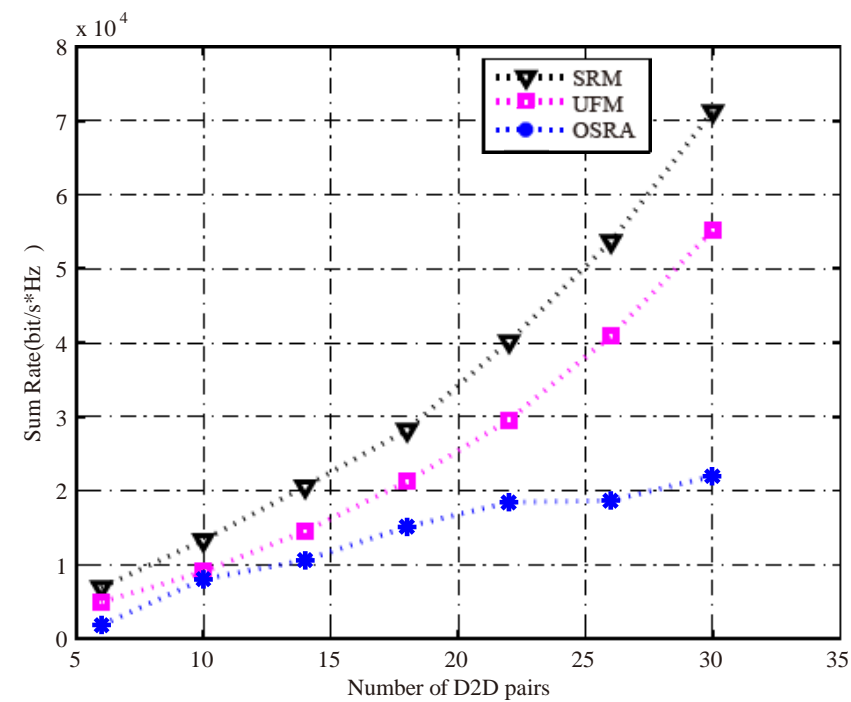

Fig. 4. Comparison of sum rate for different resource allocation algorithms, when the number of D2D users increases 


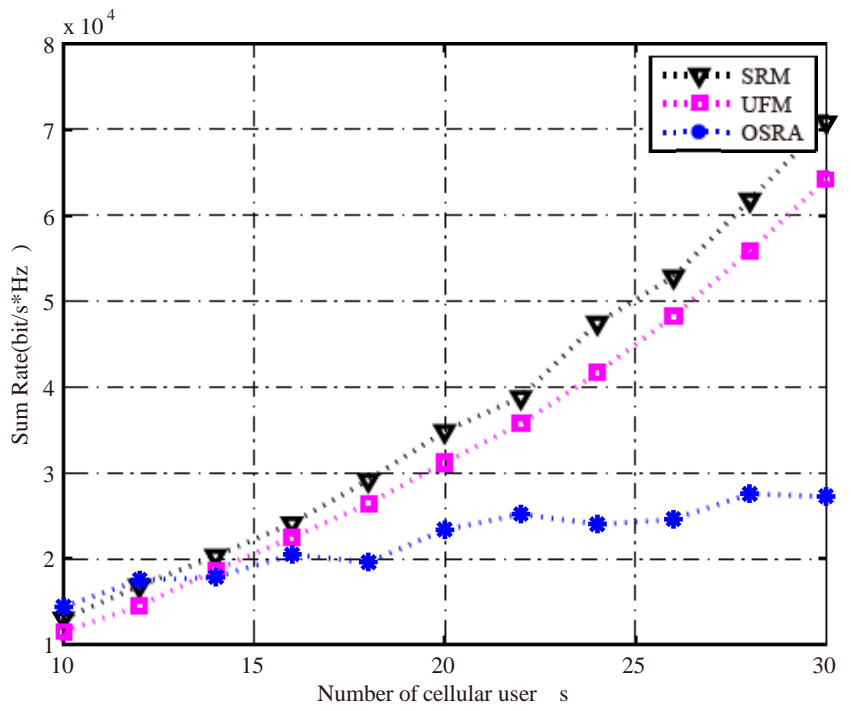

Fig. 5. Comparison of sum rate for different resource allocation algorithms, when the number of cellular users increases

For the service latency, we compare the SRM to UFM and OSRA. The simulation results are shown in the Fig. $\mathbf{6}$ and Fig. 7. When the number of cellular users increases, the total service latency in the system decreases and SRM has better performance than the other scheme. Moreover, UFM scheme has the most influence according to the cellular users. Our proposed scheme SRM has a little improvement than OSRA, which is because we estimate the social value more accurately. Next, when the number of D2D users increases in Fig. 7, the total service latency has some difference from Fig. 6. The D2D users have reduced the more latency than cellular users, which is reasonable, because D2D is an end-to-end communication. And there are a little reduction between the three scheme.

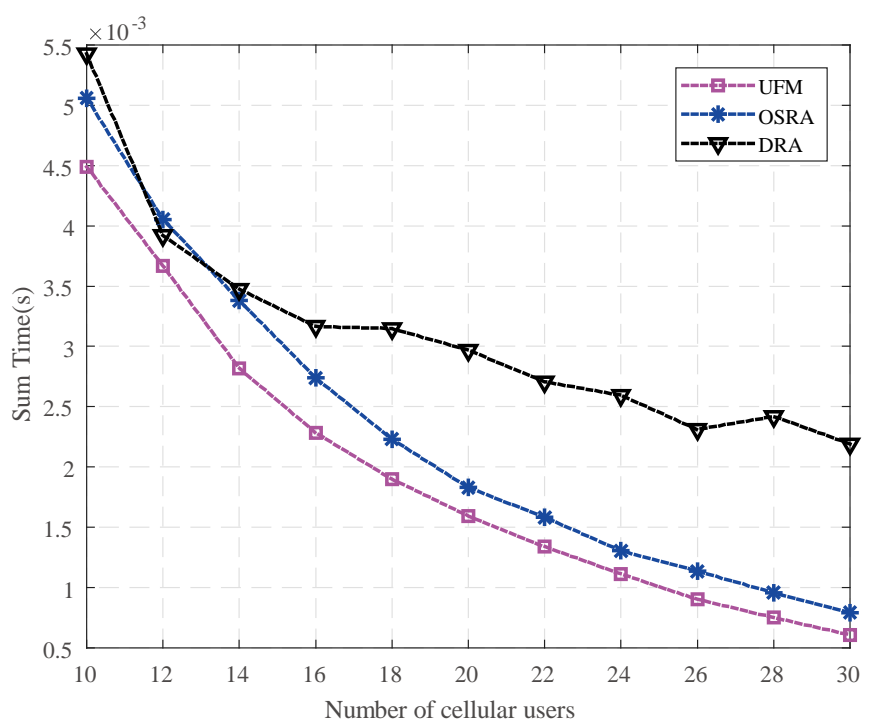

Fig. 6. Comparison of service latency for different resource allocation algorithms, when the number of cellular users increases 


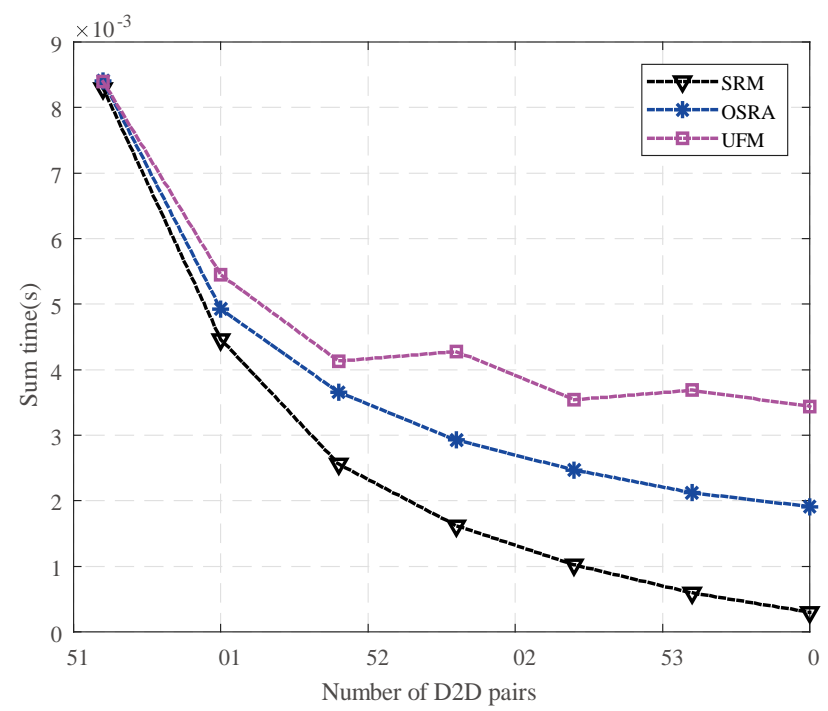

Fig. 7. Comparison of service latency for different resource allocation algorithms, when the number of D2D users increases

In Fig. 8, we have presented the relationship between interference and social utility. While the number of D2D users remains the same, the cellular users have achieved the more interference in the network, when the channels are congested. However, it drops pointedly and trend to a smooth and steady situation. Note that the social utility is only related to D2D users, so that the curve is steady. Meanwhile, it does not fluctuate very much. As shown in Fig. 9, the throughput of cellular network increases based on cellular users and it is almost a liner function. But the throughput of D2D network also stays a steady status, which means the interference of cellular users do not reduce the D2D transmission rate. Moreover, the D2D user has maintained the good social connection in the network.

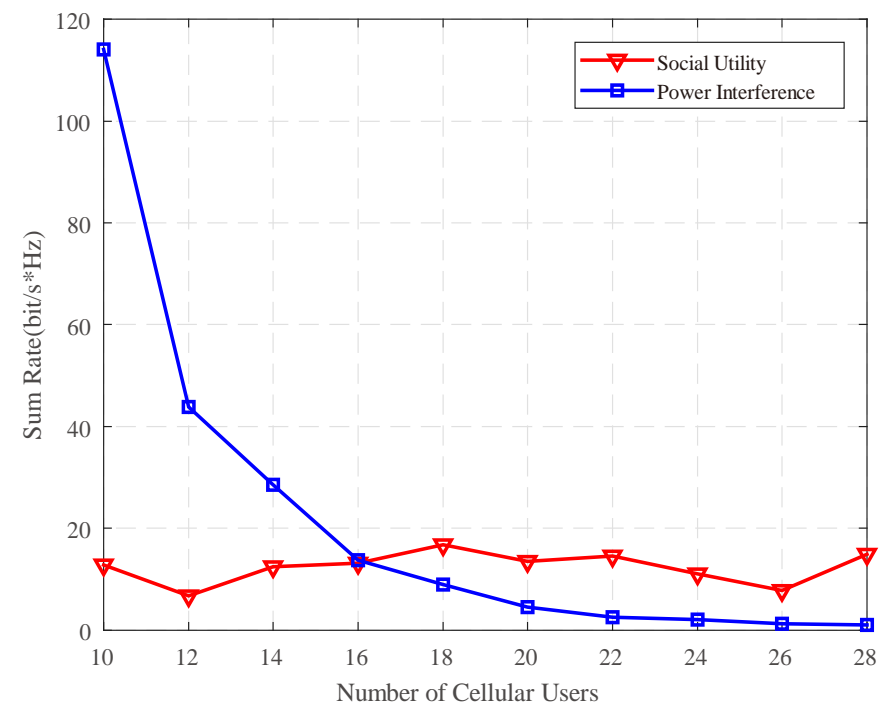

Fig. 8. Discussion of interference in the system 


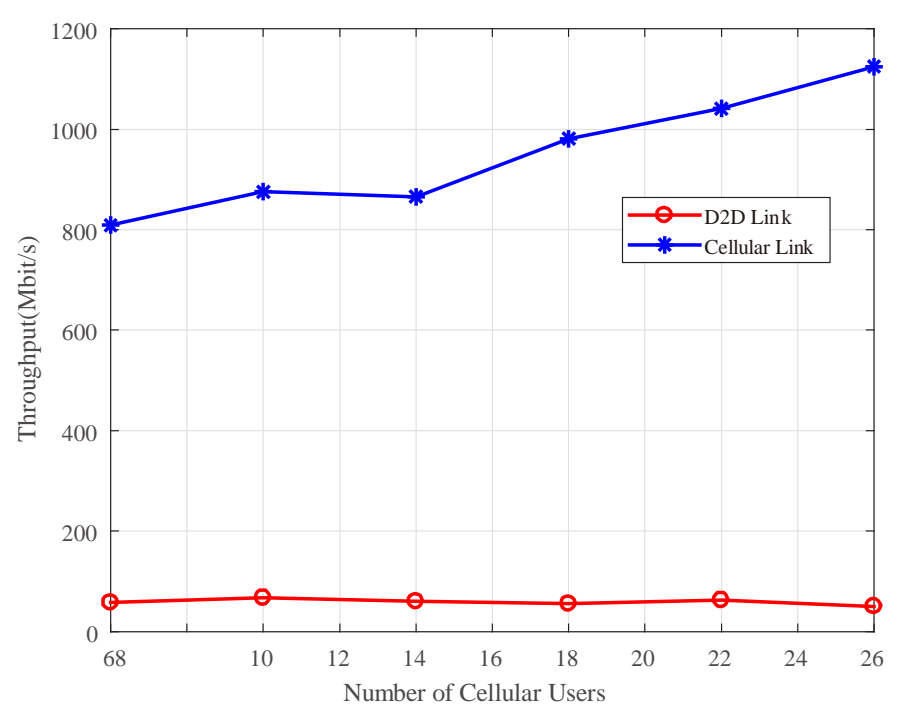

Fig. 9. Analysis of throughput for different modes in the system

\section{Conclusions and Future Work}

In this paper, we propose a social-aware resource allocation scheme in the underlay D2D communication system based on the realistic social network. By combining the social and physical characteristics, we divide the community into several clusters. We also refer to matching theory and design a rate-maximization oriented SRM resource allocation algorithm. Through the simulation result, we have demonstrated the effectiveness of our proposed SRM algorithm, suggesting that our proposed social-aware scheme is more suitable for future joint resource allocation scenarios.

\section{Acknowledgements}

This work is partly supported by the National Natural Science Foundation of China (61571240, 61671253, 61901227); the major projects of the Natural Science Foundation of the Jiangsu Higher Educations Institutions (16KJA510004, 19KJB510049); the Postdoctoral Science Foundation of China (Grant No.2017M611881); the National Engineering Research Center of Communications and Networking (Nanjing University of Posts and Telecommunications (Grant No.TXKY17003); Innovation Program of Postgraduate Research in Jiangsu Province (KYCX19_0889), Scientific Research Foundation of NUPT (NY218011, NY220007).

\section{References}

[1] L. Zhou, D. Wu, X. Wei, and Z. Dong, “Seeing Isn't Believing: QoE Evaluation for Privacy-Aware Users,” IEEE Journal on Selected Areas in Communications, vol. 37, no. 7, pp. 1656-1665, July 2019. Article (CrossRef Link)

[2] T. O. Olwal, K. Djouani and A. M. Kurien, “A Survey of Resource Management Toward 5G Radio Access Networks,” IEEE Communications Surveys and Tutorials, vol. 18, no. 3, pp. 1656-1686, third quarter 2016. Article (CrossRef Link) 
[3] M. Jo, T. Maksymyuk, B. Strykhalyuk and C. Cho, "Device-to-device-based heterogeneous radio access network architecture for mobile cloud computing,” IEEE Wireless Communications, vol. 22, no. 3, pp. 50-58, June 2015. Article (CrossRef Link)

[4] C. Xu et al., "Efficiency Resource Allocation for Device-to-Device Underlay Communication Systems: A Reverse Iterative Combinatorial Auction Based Approach,” IEEE Journal on Selected Areas in Communications, vol. 31, no. 9, pp. 348-358, Sept. 2013. Article (CrossRef Link)

[5] L. Zhou, D. Wu, J. Chen, and Z. Dong, "Greening the Smart Cities: Energy-Efficient Massive Content Delivery via D2D Communications,” IEEE Transactions on Industrial Informatics, vol. 14, no. 4, pp.1626-1634, April 2018. Article (CrossRef Link)

[6] L. Zhou, D. Wu, J. Chen, and Z. Dong, "When Computation Hugs Intelligence: Content-Aware Data Processing for Industrial IoT, “ IEEE Internet of Things Journal, vol. 5, no. 3, pp. 1657-1666, June 2018. Article (CrossRef Link)

[7] E. Ahmed, I. Yaqoob, A. Gani, M. Imran and M. Guizani, “Social-Aware Resource Allocation and Optimization for D2D Communication,” IEEE Wireless Communications, vol. 24, no. 3, pp. 122-129, June 2017. Article (CrossRef Link)

[8] O. Semiari, W. Saad, S. Valentin, M. Bennis and H. V. Poor, "Context-Aware Small Cell Networks: How Social Metrics Improve Wireless Resource Allocation,” IEEE Transactions on Wireless Communications, vol. 14, no. 11, pp. 5927-5940, Nov. 2015. Article (CrossRef Link)

[9] F. Wang, Y. Li, Z. Wang and Z. Yang, "Social-Community-Aware Resource Allocation for D2D Communications Underlaying Cellular Networks,” IEEE Transactions on Vehicular Technology, vol. 65, no. 5, pp. 3628-3640, May 2016. Article (CrossRef Link)

[10] B. Fan, H. Tian, L. Jiang and A. V. Vasilakos, “A Social-Aware Virtual MAC Protocol for Energy-Efficient D2D Communications Underlying Heterogeneous Cellular Networks,” IEEE Transactions on Vehicular Technology, vol. 67, no. 9, pp. 8372-8385, Sept. 2018. Article (CrossRef Link)

[11] Y. Gao, Y. Xiao, M. Wu, M. Xiao and J. Shao, "Dynamic Social-Aware Peer Selection for Cooperative Relay Management With D2D Communications," IEEE Transactions on Communications, vol. 67, no. 5, pp. 3124-3139, May 2019. Article (CrossRef Link)

[12] Y. Li, Z. Zhang, H. Wang and Q. Yang, “SERS: Social-Aware Energy-Efficient Relay Selection in D2D Communications," IEEE Transactions on Vehicular Technology, vol. 67, no. 6, pp. 5331-5345, June 2018. Article (CrossRef Link)

[13] D. Wu, L. Zhou, Y. Cai and Y. Qian, “Optimal Content Sharing Mode Selection for Social-Aware D2D Communications,” IEEE Wireless Communications Letters, vol. 7, no. 6, pp. 910-913, Dec. 2018. Article (CrossRef Link)

[14] Y. Gu, Y. Zhang, M. Pan and Z. Han, "Matching and Cheating in Device to Device Communications Underlying Cellular Networks," IEEE Journal on Selected Areas in Communications, vol. 33, no. 10, pp. 2156-2166, Oct. 2015. Article (CrossRef Link)

[15] D. Wu, L. Zhou, Y. Cai, H. Chao and Y. Qian, "Physical-Social-Aware D2D Content Sharing Networks: A Provider-Demander Matching Game,” IEEE Transactions on Vehicular Technology, vol. 67, no. 8, pp. 7538-7549, Aug. 2018. Article (CrossRef Link)

[16] H. Hu, Y. Wen, T. Chua, J. Huang, W. Zhu and X. Li, "Joint Content Replication and Request Routing for Social Video Distribution Over Cloud CDN: A Community Clustering Method,” IEEE Transactions on Circuits and Systems for Video Technology, vol. 26, no. 7, pp. 1320-1333, July 2016. Article (CrossRef Link)

[17] Z. Su, Y. Hui and S. Guo, "D2D-based content delivery with parked vehicles in vehicular social networks,” IEEE Wireless Communications, vol. 23, no. 4, pp. 90-95, August 2016. Article (CrossRef Link)

[18] Z. Wang, L. Sun, M. Zhang, H. Pang, E. Tian and W. Zhu, "Propagation- and Mobility-Aware D2D Social Content Replication,” IEEE Transactions on Mobile Computing, vol. 16, no. 4, pp. 1107-1120, April 2017. Article (CrossRef Link) 
[19] D. Wu, L. Zhou and Y. Cai, "Social-Aware Rate Based Content Sharing Mode Selection for D2D Content Sharing Scenarios,” IEEE Transactions on Multimedia, vol. 19, no. 11, pp. 2571-2582, Nov. 2017. Article (CrossRef Link)

[20] P. Zhao, L. Feng, P. Yu, W. Li and X. Qiu, "A Social-Aware Resource Allocation for 5G Device-to-Device Multicast Communication,” IEEE Access, vol. 5, pp. 15717-15730, 2017. Article (CrossRef Link)

[21] T. Wang, Y. Sun, L. Song and Z. Han, "Social Data Offloading in D2D-Enhanced Cellular Networks by Network Formation Games,” IEEE Transactions on Wireless Communications, vol. 14, no. 12, pp. 7004-7015, Dec. 2015. Article (CrossRef Link)

[22] E. Ahmed, I. Yaqoob, A. Gani, M. Imran and M. Guizani, "Social-Aware Resource Allocation and Optimization for D2D Communication,” IEEE Wireless Communications, vol. 24, no. 3, pp. 122-129, June 2017. Article (CrossRef Link)

[23] Y. Zhao, Y. Li, Y. Cao, T. Jiang and N. Ge, "Social-Aware Resource Allocation for Device-to-Device Communications Underlaying Cellular Networks," IEEE Transactions on Wireless Communications, vol. 14, no. 12, pp. 6621-6634, Dec. 2015. Article (CrossRef Link)

[24] F. Wang, Y. Li, Z. Wang and Z. Yang, "Social-Community-Aware Resource Allocation for D2D Communications Underlaying Cellular Networks,” IEEE Transactions on Vehicular Technology, vol. 65, no. 5, pp. 3628-3640, May 2016. Article (CrossRef Link)

[25] K. Wu, M. Jiang and H. Tan, “D2D Relay Selection Based on Joint Fuzzy and Entropy Theories With Social Similarity,” IEEE Transactions on Vehicular Technology, vol. 67, no. 9, pp. 8796-8807, Sept. 2018. Article (CrossRef Link)

[26] Y. Gao, Y. Xiao, M. Wu, M. Xiao and J. Shao, "Dynamic Social-Aware Peer Selection for Cooperative Relay Management With D2D Communications," IEEE Transactions on Communications, vol. 67, no. 5, pp. 3124-3139, May 2019. Article (CrossRef Link)

[27] X. Chen, B. Proulx, X. Gong and J. Zhang, "Exploiting Social Ties for Cooperative D2D Communications: A Mobile Social Networking Case,” IEEE/ACM Transactions on Networking, vol. 23, no. 5, pp. 1471-1484, Oct. 2015. Article (CrossRef Link)

[28] Y. Li, Z. Zhang, H. Wang and Q. Yang, “SERS: Social-Aware Energy-Efficient Relay Selection in D2D Communications," IEEE Transactions on Vehicular Technology, vol. 67, no. 6, pp. 5331-5345, June 2018. Article (CrossRef Link)

[29] H. Hu, Y. Wen, H. Luan, T. Chua and X. Li, "Toward Multiscreen Social TV with Geolocation-Aware Social Sense,” IEEE Multimedia, vol. 21, no. 3, pp. 10-19, July-Sept. 2014. Article (CrossRef Link)

[30] M. Chen, L. Wang, J. Chen, X. Wei and L. Lei, “A Computing and Content Delivery Network in the Smart City: Scenario, Framework, and Analysis,” IEEE Network, vol. 33, no. 2, pp. 89-95, March/April 2019. Article (CrossRef Link) 


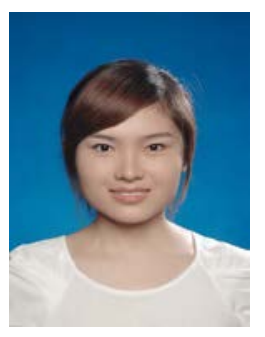

Wenqin Zhuang received her M.Sc. degree with major on Electromagnetic Field and Microwave Technology from the Nanjing University of Posts and Telecommunications, Nanjing, China, in 2012. She is currently a technician and Ph.D. candidate in Nanjing University of Posts and Telecommunications. Her current research interests include wireless multimedia communication, image processing, and Quality of Experience (QoE) of multimedia distribution.

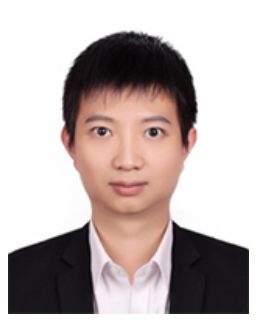

Mingkai Chen received his Ph.D. degree in information and communication engineering from Nanjing University of Posts and Telecommunications, China in 2019. He is currently a lecturer at Nanjing University of Posts and Telecommunications, China. His research interests are in the area of multimedia communications and computing, resource allocation, and signal processing in wireless networks.

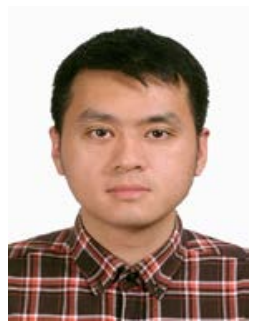

Xin Wei received his Ph.D. degree major at Information and Communication Engineering from Southeast University, Nanjing, China in 2009. Now, he is an associate professor in Nanjing University of Posts and Telecommunications, China. His research interests are in the area of multimedia communications, machine learning.

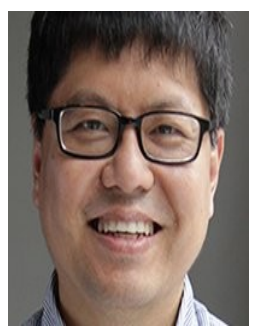

Haibo Li is a professor in Nanjing University of Posts and Telecommunications, working in the fields of computational imaging and computer vision. 